\title{
Integrated analysis of IncRNA, miRNA and mRNA expression profiling in patients with systemic lupus erythematosus
}

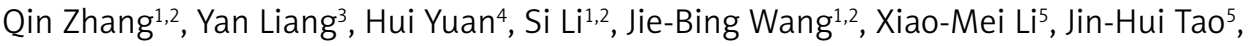 \\ Hai-Feng Pan ${ }^{1,2}$, Dong-Qing Ye ${ }^{1,2}$
}

\begin{abstract}
1Department of Epidemiology and Biostatistics, School of Public Health, Anhui Medical University, Hefei, Anhui, China

${ }^{2}$ Anhui Province Key Laboratory of Major Autoimmune Diseases, Hefei, Anhui, China ${ }^{3}$ Department of Nephrology, the First Affiliated Hospital, Sun Yat-sen University, Guangzhou, Guangdong, China

${ }^{4}$ Department of Preventive Medicine, Wannan Medical College, Wuhu, Anhui, China ${ }^{5}$ Department of Rheumatology and Immunology, Anhui Provincial Hospital, Hefei, Anhui, China
\end{abstract}

Submitted: 10 July 2018

Accepted: 11 September 2018

Arch Med Sci 2019; 15 (4): 872-879

DOI: https://doi.org/10.5114/aoms.2018.79145

Copyright $\odot 2018$ Termedia \& Banach

\section{Abstract}

Introduction: A great deal of research has reported dysregulated expression of genes in systemic lupus erythematosus (SLE). This study aimed to analyze the IncRNA, miRNA and mRNA expression profile in SLE.

Material and methods: RNA sequencing (RNA-seq) was used to detect the dysregulated RNAs in SLE. Gene Ontology (GO) and Kyoto Encyclopedia of Genes and Genomes (KEGG) pathways analysis were used to explore the function of these differentially expressed RNAs.

Results: 2,353 IncRNAs, 827 mRNAs and 24 miRNAs were shown to be differentially expressed. GO analyses demonstrated that differentially expressed RNAs were enriched in a variety of molecular functions and biological processes including ribonucleotide, protein serine/threonine kinase activity function, regulation of $B$ cell differentiation and others. KEGG pathway analyses revealed that differentially expressed mRNAs and IncRNAs were both enriched in FcyR-mediated phagocytosis, glycosaminoglycan biosynthesis-chondroitin sulfate/dermatan sulfate and glyoxylate and dicarboxylate metabolism pathways. The up-regulated miRNAs target genes were mainly enriched in the nuclear factor- $\mathrm{KB}(\mathrm{NF}-\mathrm{\kappa B})$ signaling pathway. The down-regulated miRNAs target genes were significantly enriched in metabolism of xenobiotics by cytochrome P450, bile secretion and terpenoid backbone biosynthesis pathways.

Conclusions: The current study reveals a comprehensive expression profile of IncRNAs, miRNAs and mRNAs and implies potential regulatory functions of these RNAs which are involved in the pathogenesis of SLE.

Key words: IncRNA, miRNA, mRNA, RNA-seq, systemic lupus erythematosus.

\section{Introduction}

Systemic lupus erythematosus (SLE), a kind of severe autoimmune disease (AID), is characterized by loss of immunological tolerance to self-nuclear antigens, abnormal T- and B-cell responses and autoanti-

\author{
Corresponding authors: \\ Dong-Qing Ye, \\ Hai-Feng Pan \\ Department \\ of Epidemiology \\ and Biostatistics \\ School of \\ Public Health \\ Anhui Medical University \\ 81 Meishan Road \\ Hefei, Anhui \\ 230032 China \\ Phone: +8655165161165 \\ Fax: +8655165161171 \\ E-mail: ydq@ahmu.edu.cn, \\ ydqahmu@126.com \\ panhaifeng@ahmu.edu.cn, \\ panhaifeng1982@sina.com
}


body production $[1,2]$. To date, the pathogenesis of SLE remains uncertain [3]. Particularly much remains to be illuminated about gene expression levels and related regulatory mechanisms in SLE. Nowadays, emergence of transcriptomics shift the trend toward genome-wide expression, and becomes an important method to identify the gene signatures and biological function. In the past, extensive studies about transcriptomics mainly paid attention to protein-coding genes. Recently, studies about transcriptomics began to focus on noncoding RNA (ncRNA) transcripts. ncRNAs are RNA molecules which are not translated into proteins [4]. Long ncRNA (IncRNA) and micro RNA (miRNA) are two major types of ncRNAs, and as crucial regulators can be found in various biological processes by interfering with gene expression [5].

LncRNA is larger than 200 nt in length. Recently, many studies have demonstrated the important role of IncRNAs in the immune system, including dendritic cell differentiation, $T$ cell activation and granulocytic differentiation, etc. [6, 7]. But studies on the role of IncRNAs in SLE are very limited. Growth arrest specific 5 (GAS5) was reported to be connected with increased susceptibility to SLE in a mouse model; linc0597 and linc0949 were found significantly decreased in SLE patients [8-10]. In our recent study, linc-DC and GAS5 were decreased and linc0597 overexpressed in plasma of SLE patients, and linc0597, GAS5 and Inc-DC can be used as potential biomarkers for SLE [11]. miRNAs range from 18 to $25 \mathrm{nt}$ in length and regulate approximately $90 \%$ of protein-coding genes [12]. In previous studies, many miRNAs such as miR-155, miR-17, miR-181b, miR-142-3p and miR-326 were reported to be related to the pathogenesis of SLE [13-15]. However, there are no studies investigating mRNAs, InCRNAs and miRNAs expression simultaneously in SLE.

As a revolutionary tool for transcriptomic study, RNA sequencing (RNA-seq), the first sequencingbased method, can act as a high-throughput and quantitative survey of the complete set of transcripts; therefore it has become an important and widely used method for transcriptomic research $[16,17]$. Recently, RNA-seq has also been used in several studies of SLE pathogenesis [18, 19].

In the present study, to identify the integrated expression profile of IncRNA, miRNA and mRNA in SLE, RNA-seq was performed. In addition, to describe the underling biological functions of differentially expressed RNAs, bioinformation analysis was conducted.

\section{Material and methods}

\section{Subjects}

All participants in our study were in accordance with the ethical standards of the institutional and/ or national research committee, as well as the 1964 Helsinki declaration and its later amendments or comparable ethical standards. Informed consent from all participants in our study was also collected. The SLE patients were recruited from Anhui Provincial Hospital and the First Affiliated Hospital of Anhui Medical University. The age- and sexmatched healthy controls $(\mathrm{HC})$ were recruited from the Physical Examination Center of the First Affiliated Hospital of Anhui Medical University. Finally our study included 24 SLE patients and 24 HC. The inclusion criteria were according to the SLE classification of the American College of Rheumatology in 1997; all patients must fulfill at least four of the criteria [20]. A questionnaire survey was used to collect demographic data and clinical manifestations of all patients. Laboratory data were obtained from the medical record. The SLE disease activity index (SLEDAI) was used to evaluate disease activity of SLE patients [21]. Active SLE was defined as a SLEDAI score $\geq 8$. Clinical manifestations and laboratory records of all subjects are shown in Table I.

\section{Blood samples}

Blood samples $(5 \mathrm{ml})$ were harvested directly from all participants. All blood samples were drawn by venepuncture and collected into PAXgene Blood RNA tubes for transcriptomics assays, and allowed to stand at room temperature for $2 \mathrm{~h}$, then frozen at $-20^{\circ} \mathrm{C}$ overnight, and then stored at $-80^{\circ} \mathrm{C}$. Equal amounts of sample from 8 different individuals (SLE patients and HC group) were randomly pooled to minimize the biological variation. In total, there were three samples in the SLE group, and three in the $\mathrm{HC}$ group.

\section{Transcriptomics analysis of blood samples (RNA sequencing, RNA-seq)}

$m R N A$ Prior to library preparation, the concentration and integrity were detected for each sample by an Agilent 2,100 Bioanalyzer (Agilent RNA 6000 Nano Kit).

Firstly, total RNA was extracted from mixed samples of with equal weights. Subsequently, Oligo-dT beads were used to purify the total RNA sample (200 ng), then poly (A)-containing mRNA was fragmented into small pieces with elute, prime and fragment mix. First strand CDNA was then synthesized via the cleavage of short mRNA fragments. Then the second-strand cDNA was synthesized, and the cDNA fragments were subjected to end repair. After end-repair and adapter-ligation, the products were amplified through PCR and purified to construct the CDNA library.

The library preparations were sequenced on an Illumina Hiseq 2000 platform; read length 90 nt is the most common sequencing strategy. 
Qin Zhang, Yan Liang, Hui Yuan, Si Li, Jie-Bing Wang, Xiao-Mei Li, Jin-Hui Tao, Hai-Feng Pan, Dong-Qing Ye

Table I. Demographics, clinical manifestations and laboratory characteristics of subjects

\begin{tabular}{|c|c|c|}
\hline Parameter & SLE group $(n=24)$ & Healthy controls $(n=24)$ \\
\hline \multicolumn{3}{|l|}{ Demographics: } \\
\hline Sex, female/male, $n$ & $24 / 0$ & $24 / 0$ \\
\hline Age, median (range) [years] & $30.5(16-50)$ & $29.5(20-55)$ \\
\hline SLEDAI score, median (range) & $11(4-28)$ & - \\
\hline \multicolumn{3}{|l|}{ Clinical manifestations, $n$ (\%): } \\
\hline Nephritis & $13(54)$ & - \\
\hline Mucocutaneous manifestations & $12(50)$ & - \\
\hline Serositis & $6(25)$ & - \\
\hline Arthritis & $5(21)$ & - \\
\hline Neuropsychiatric manifestations & $2(8)$ & - \\
\hline Vasculitis & $2(8)$ & - \\
\hline Myositis & $1(4)$ & - \\
\hline Laboratory characteristics: & & - \\
\hline Anti-Sm & $8(33)$ & - \\
\hline Anti-SSA/Ro & $17(71)$ & - \\
\hline Anti-SSB/La & $7(29)$ & - \\
\hline Anti-RNP & $10(42)$ & - \\
\hline Anti-Rib P & $8(33)$ & - \\
\hline Anti-dsDNA & $10(42)$ & - \\
\hline Thrombocytopenia & $11(45)$ & - \\
\hline Leukopenia & $10(42)$ & - \\
\hline Low complement C3 & $22(92)$ & - \\
\hline Low complement C4 & $16(67)$ & - \\
\hline High ESR & $21(88)$ & - \\
\hline High CRP & $13(54)$ & - \\
\hline Immunosuppressive treatments & $9(38)$ & - \\
\hline
\end{tabular}

LncRNA Ribo-Zero Magnetic Kit (Human/ Mouse/Rat) (Epicentre) was used to deplete rRNA from $5 \mu \mathrm{g}$ of total RNA. The retrieved RNA was fragmented by adding First Strand Master Mix. The library preparations were the same as mRNA.

miRNA Approximately $1 \mu \mathrm{g}$ RNA from each sample was used to construct the subsequent cDNA library following the protocol of TruSeq Small RNA Sample Prep Kits. RNAs were ligated to $3^{\prime}$ and $5^{\prime}$ adaptor sequentially, reverse transcribed to CDNA and PCR amplified. Then the PCR products were purified with PAGE gel after ethanol precipitation and washing, and then the purified small RNA libraries were quantified and used for cluster generation and 36 nt single end library preparations were sequenced on the Illumina Hiseq 2000 platform.

\section{Statistical analysis}

Data were expressed as $(\bar{x} \pm s)$ or medians with range. Comparison of continuous data was performed by independent Student's $t$-test. The Statistical Package for the Social Sciences (SPSS) 23.0 for Windows (IBM Co., Armonk, NY, USA) was used for statistical analyses. $P$-values $<0.05$ were considered statistically significant.

Sequencing data were analyzed by using fold changes (FC) and Student's t-test. $|g| F C \mid \geq 2.0$ and $P_{\text {adj }} \leq 0.05$ were set as the criterion to identify the differentially expressed mRNAs and IncRNAs. And $|\mathrm{g}| \mathrm{FC} \mid \geq 1.0$ and $P_{\text {adj }} \leq 0.3$ were set as the threshold to identify the aberrantly expressed miRNAs. 


\section{Functional group analysis}

The filtered co-expressed genes were defined as potential target genes of IncRNAs. Pearson and Spearman correlations were calculated for each pair of genes and the correlation coefficient $\geq 0.6$ as our criterion.

We used the miRTarBase to predict the potential targets of those differentially expressed miRNAs miRTarBase (http://mirtarbase.mbc.nctu. edu.tw) is a database that provides the latest and extensive validated miRNA-target interaction information [22].

To determine the division of SLE relevant and irrelevant RNAs, we conducted the Gene Ontology (GO) terms and Kyoto Encyclopedia of Genes and Genomes (KEGG) pathways analysis.

GO (http://www.geneontology.org) analysis was used to detect the function of that associates distinctively expressed genes with GO terms and reveals gene regulatory networks on the basis of cellular components, biological processes and molecular functions [23, 24]. The hypergeometric test was used to get the target GO terms. If the $p$-value is less than 0.05 , the GO term is significant enrichment in differentially expressed RNAs. KEGG (http://www.genome.jp/kegg/) analysis is used to identify the pathways of these differentially expressed RNAs enriched by Fisher's exact test $[25,26]$. The KEGG analysis was the same as GO enrichment. If the $P_{\text {adj }}$ is less than 0.05 , the pathway is significant enrichment in differentially expressed RNAs.

\section{Results}

\section{Summary of RNA-seq}

Deep sequencing generated the mean number of raw reads, 273,119,891.3 and 266,323,616.7, for SLE patients and the HC group, respectively. Removing reads with non-canonical letters or with low quality, and discarding the sequences shorter than $18 \mathrm{nt}$, the mean numbers of clean reads were 259,455,621.3 and 252,555,396.0, for SLE patients and the $\mathrm{HC}$ group, respectively, which remained for analysis (Table II). The filtered clean reads has been submitted to the American National Center for Biotechnology Information (Num: SRP076773). A total of 93,267 transcripts were detected in the current study, in which 37,637 transcripts were identified as mRNA and 55,630 transcripts as IncRNA.

\section{Differentially expressed IncRNAs and mRNAs}

In total 2,354 IncRNAs and 827 mRNAs were identified to be significantly expressed. Among them, 1,873 known IncRNAs (221 up-regulated and 1,652 down-regulated) were identified in SLE patients. Additionally, 481 novel IncRNAs (73 increased and 408 decreased) were revealed (Supplementary Tables SI, SII). We also found that a total of 78 known mRNAs and 2 novel mRNAs increased, and 729 known mRNAs and 18 novel mRNAs decreased in the SLE patients (Supplementary Tables SIII, SIV, Figures 1, 2).

\section{mRNAs GO and KEGG analyses}

GO analysis was used to explore the function of the significantly altered genes (Supplementary Table SV). The differentially expressed mRNAs were mainly enriched in nine molecular function terms containing ribonucleotide, purine nucleotide, and protein serine/threonine kinase activity, and one biological process term of a cellular metabolic process.

The KEGG pathway analysis was performed to identify pathways which were significantly enriched with differentially expressed RNAs ( $p<$ 0.05). In total, differentially expressed mRNAs significantly enriched 16 pathways (Supplementary Table SVI). Among these pathways, the organismal systems pathways category contained the largest number of differentially expressed genes and the FcyR-mediated phagocytosis pathway contained the most differentially expressed genes.

\section{GO and pathway analysis of differentially expressed IncRNA-targeted mRNA}

Since the function of IncRNAs is mainly executed in protein-coding target genes, we utilized

Table II. Sequence comparison results of RNA-seq

\begin{tabular}{|lcccccccc|}
\hline Samples & $\begin{array}{c}\text { Total raw } \\
\text { reads }\end{array}$ & $\begin{array}{c}\text { Total clean } \\
\text { reads }\end{array}$ & $\begin{array}{c}\text { Total rRNA } \\
\text { filter reads }\end{array}$ & $\begin{array}{c}\text { Total base } \\
\text { filter reads }\end{array}$ & $\begin{array}{c}\text { Reads with } \\
\text { adapter }\end{array}$ & $\begin{array}{c}\text { Reads with } \\
\text { low quality }\end{array}$ & $\begin{array}{c}\text { Reads with } \\
\text { duplica- } \\
\text { tions }\end{array}$ & $\begin{array}{c}\text { Read with } \\
\text { N rate } \\
\text { exceed }\end{array}$ \\
\hline SLE_1 & 282577972 & 267811334 & 10115522 & 4651116 & 4536538 & 54 & - & - \\
\hline SLE_2 & 265576840 & 252259496 & 9079520 & 4237824 & 4128966 & 50 & 114524 \\
\hline SLE_3 & 271204862 & 258296034 & 8591074 & 4317754 & 4206328 & 34 & - & 111392 \\
\hline HC_1 & 269855160 & 259507288 & 8324268 & 2023604 & 1912514 & 46 & - & 111044 \\
\hline HC_2 & 256683874 & 240920194 & 10251286 & 5512394 & 5409028 & 46 & - & 103320 \\
\hline HC_3 & 272431816 & 257238706 & 11357320 & 3835790 & 3725938 & 42 & - & 109810 \\
\hline
\end{tabular}




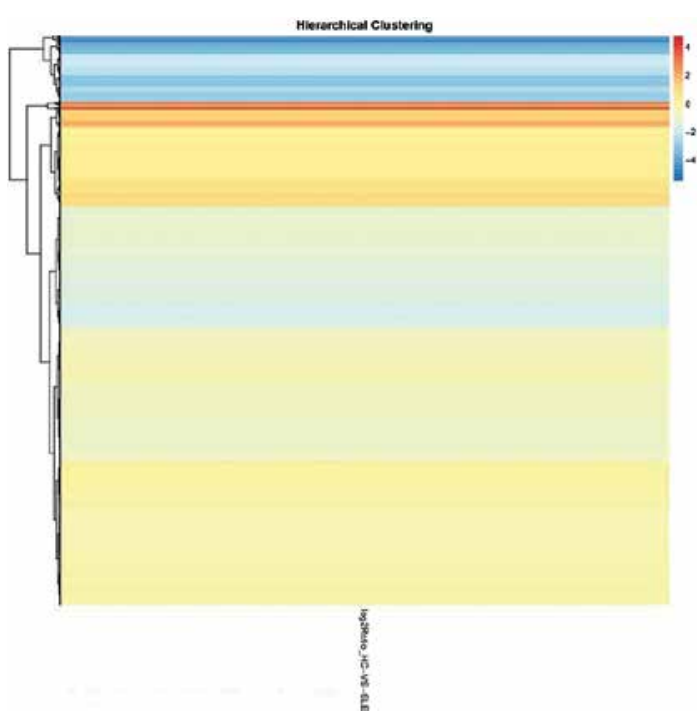

Figure 1. Differentially expressed known RNAs between SLE and HC. Hierarchical analysis of known RNAs that were differentially expressed between two groups

the co-expression patterns of mRNAs and IncRNAs to infer biological functions for the latter. These genes were considered as potential target genes of IncRNAs. We then used GO and KEGG analyses to identify the inferred functions about the differentially expressed IncRNAs' target genes between SLE patients and the HC group. At last, ten GO terms in cell component and five in molecular function were significantly enriched. These five GO terms in molecular function were ion binding, cation binding, metal ion binding, transition metal ion binding and zinc ion binding (Supplementary Table SVII).

The pathway analysis of IncRNAs was also conducted. These differentially expressed IncRNA-targeted mRNAs were significantly enriched in ten pathways in our study (Supplementary Table SVIII). Among them, the environmental information processing pathways category contained the largest number of differentially expressed genes, which

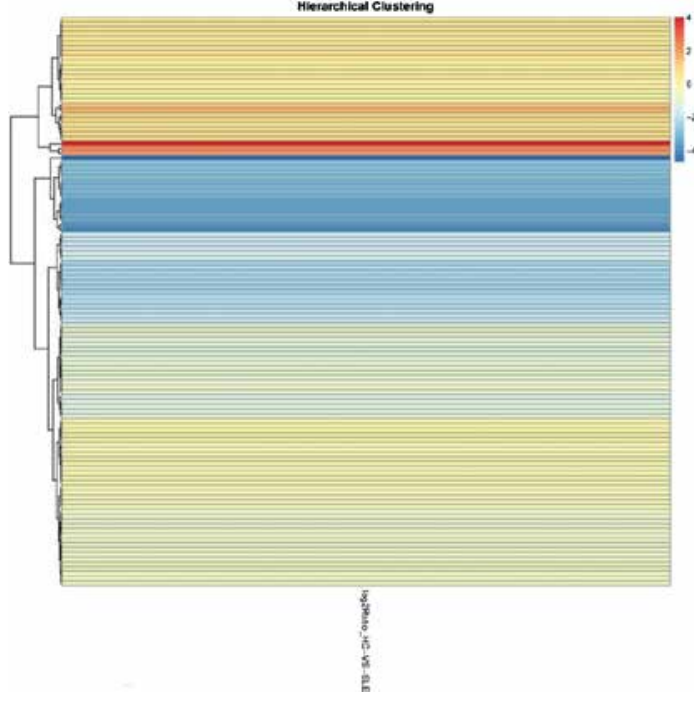

Figure 2. Differentially expressed novel RNAs between SLE and HC. Hierarchical analysis of novel RNAs that were differentially expressed between two groups

fell into two major subgroups involved in the phospholipase D signaling pathway and CAMP signaling pathway. And the results of KEGG pathway analysis of mRNA showed that the FcyR-mediated phagocytosis pathway, glycosaminoglycan biosynthesis-chondroitin sulfate/dermatan sulfate and glyoxylate and dicarboxylate metabolism were also significantly enriched.

\section{miRNA-seq summary}

The mean numbers of raw reads were generated from the deep sequencing of the small RNA libraries, 11,925,626 and 11,933,904, for SLE patients and the HC group, respectively. The mean number of clean reads, 11,548,206 and $11,518,853$, for SLE patients and the HC group, respectively, remained for analysis (Table III). Length distribution of sequenced miRNAs ranges from 15 to $30 \mathrm{nt}$, with a sharp peak at 20-24 nt.

Table III. Sequence comparison results miRNA-seq

\begin{tabular}{|lccccccccc|}
\hline Samples & $\begin{array}{c}\text { Total raw } \\
\text { reads }\end{array}$ & $\begin{array}{c}\text { Total } \\
\text { clean } \\
\text { reads }\end{array}$ & $\begin{array}{c}\text { Reads } \\
\text { with high } \\
\text { quality }\end{array}$ & $\begin{array}{c}\text { Reads } \\
\text { with 3' } \\
\text { adapter }\end{array}$ & $\begin{array}{c}\text { Reads } \\
\text { with 5' } \\
\text { adapter } \\
\text { contami- } \\
\text { nants }\end{array}$ & $\begin{array}{c}\text { Reads } \\
\text { smaller } \\
\text { than } \\
18 \text { nt }\end{array}$ & $\begin{array}{c}\text { Reads } \\
\text { with } \\
\text { insert }\end{array}$ & $\begin{array}{c}\text { Reads } \\
\text { with poly } \\
\text { A }\end{array}$ & $\begin{array}{c}\text { Total raw } \\
\text { reads }\end{array}$ \\
\hline SLE_1 & 12010209 & 11457585 & 11987118 & 136496 & 10959 & 296431 & 85635 & 12 & 12010209 \\
\hline SLE_2 & 11585370 & 11297716 & 11564588 & 69377 & 4650 & 162011 & 30829 & 5 & 11585370 \\
\hline SLE_3 & 12181299 & 11889318 & 12159618 & 69402 & 2456 & 182301 & 16139 & 2 & 12181299 \\
\hline HC_1 & 11746477 & 11329762 & 11723513 & 71317 & 9346 & 217016 & 96070 & 2 & 11746477 \\
\hline HC_2 & 12391342 & 11849986 & 12367782 & 68437 & 13059 & 329485 & 106811 & 4 & 12391342 \\
\hline HC_3 & 11663894 & 11376811 & 11640824 & 53184 & 1723 & 198184 & 10917 & 5 & 11663894 \\
\hline
\end{tabular}




\section{Differentially expressed miRNA}

In the miRNA sequencing study, 24 miRNAs were differentially expressed. Among these miRNAs, there were 6 increased and 18 decreased miRNAs in SLE patients (Supplementary Table SIX). hsa-miR-144-5p, hsa-miR-1304-3p and hsa-miR-30b-3p were the most significantly down-regulated miRNAs, while hsa-miR-125b-5p, hsa-miR-1226-3p and hsa-miR-618 were the most significantly up-regulated miRNAs in the SLE group.

\section{GO and pathway analysis of differentially expressed miRNA-targeted mRNA}

To provide clues for the possible roles of 24 differentially expressed miRNAs, miRTarBase were used to conduct target prediction analysis.

First we performed GO analysis based on the set of target genes (Supplementary Tables SX, $\mathrm{SXI}$ ). The results of $\mathrm{GO}$ analysis suggested that the main molecular function of up-regulated miRNAs was ankyrin binding; there were no enriched items in cell component and biological process. The main biological processes of downregulated miRNAs involvement were cell differentiation, proliferation and activation; these cells included B cells, leukocytes, lymphocytes and mononuclear cells. All these cells play important roles in the immune system. Also one GO term in cell component and three in molecular function were significantly enriched.

Then the target predicted genes were grouped into different pathways. The up-regulated miRNAs were grouped into 52 pathways and 4 pathways were significantly enriched (Supplementary Table SXII). Among these pathways, the NF- $\kappa B$ signaling pathway played an important role in the immune system. The down-regulated miRNAs were grouped into 30 pathways and 3 pathways were significantly enriched, including metabolism of xenobiotics by cytochrome P450, bile secretion and terpenoid backbone biosynthesis pathways (Supplementary Table SXIII).

\section{Discussion}

Genome-wide association studies (GWAS) have shown some genetic risk factors for AIDs, and several genes may suggest common mechanisms that lead to the development and progression of AIDs, including SLE $[27,28]$. In the past many studies have focused on RNAs, and aberrant expression of $m R N A$ levels has been observed in SLE patients, such as IL-28RA, DNMT1 and DNMT3A $[29,30]$. Recently, studies showed that ncRNAs can serve as important modulators in the development of AIDs [31]. However, the role of ncRNAs in the pathogenesis and development of SLE is still less understood. In the present study according to comparative analyses of the RNA-seq data, a total of 78 protein-coding RNAs were increased while 729 were decreased. Also, a total of 1,873 known IncRNAs (221 up-regulated and 1,652 down-regulated) and 481 novel IncRNAs (73 increased and 408 decreased) were significantly altered in SLE patients. Compared with a previous study, numerous differentially expressed RNAs in our study have been shown to play important roles in the pathogenesis of SLE, including receptor of IL-21 $(I L-21 R)$ [32], receptor of IL-23 (IL-23R) [33] and toll-like receptors 2 (TLR2) [34], etc. In addition, several differentially expressed genes such as Yippee-like-4 (YPEL4), Ubiquilin-4 (UBQLN4), interferon regulatory factor- 6 (IRF6) and other genes were reported first in our study.

Previous studies have reported that miRNAs were critical for not only the development of the immune system, but also for the function of both innate and adaptive arms [35]. We found that 6 miRNAs were increased and 18 miRNAs were decreased. Among these differentially expressed miRNAs, hsa-let-7a-5p [36], hsa-miR-125b [37] and hsa-miR-148a [38] have been reported to connect with SLE. In addition, we identified several differentially expressed miRNAs that have not been reported previously in SLE, such as hsa-miR144-5p, hsa-miR-1304-3p, hsa-miR-30b-3p.

Precisely annotating the functions of RNAs remains complex. In this study, according to the results of GO analysis, differentially expressed mRNAs were mainly involved in the molecular function terms of ribonucleotide, purine nucleotide, and protein serine/threonine kinase activity. Protein serine/threonine kinase activity is a major component of apoptotic factor. A previous study has shown that serine/threonine-protein kinase 17A (SKT17A) was associated with SLE susceptibility [39]. LncRNAs cover nearly all aspects of gene expression and protein translation. The main function of up-regulated miRNAs was ankyrin binding. The main biological process of down-regulated miRNA involvement was cell differentiation, proliferation and activation; these cells include B cells, leukocytes, lymphocytes and mononuclear cells. Especially the critical role of B-cells in the pathogenesis of SLE is well recognized - from producing autoantibody to abnormal regulation of the immune system [40].

Differentially expressed mRNAs and IncRNAs were both enriched in FcyR-mediated phagocytosis, glycosaminoglycan biosynthesis-chondroitin sulfate/dermatan sulfate and glyoxylate and dicarboxylate metabolism pathways. The FcyR-mediated phagocytosis pathway belongs to the immune pathways and contained the most differentially expressed genes, such as receptor for immunoglobulin $G(F C G R)-1 B$ and YPEL4. FCGRs play an important role in linking the humoral and cellular 
portions of the immune system [41]. When bound and clustered by immune complexes, FCGRs initiate a number of cellular processes aimed at the elimination of antigen. The FCGR gene family (FCGR2A, FCGR2B, and FCGR2C) was considered to be associated with the susceptibility to SLE [42]. YPEL4 is one of the important molecules in regulating cell proliferation [43]. However, currently, the molecular mechanisms of YPEL4 in SLE remain unclear. Previous studies showed that the pathway is associated with some other AIDs, such as RA and neuromyelitis optica $[44,45]$, but did not find the FcyR-mediated phagocytosis pathway in SLE.

The results of miRNA KEGG analysis showed that some up-regulated miRNAs were significantly enriched in the NF- $\kappa B$ signaling pathway, which plays an important role in the immune system. $\mathrm{NF}-\kappa \mathrm{B}$ is a kind of transcription factors which can regulate genes involved in inflammation, cell survival and immunity, and our previous study supported the important role of the NF-кB signaling pathway in the genetic basis of SLE [46]. Our results may contribute to future genetic studies in SLE pathogenesis and may promote the development of new therapeutic strategies by targeting these pathways.

Several limitations of our study should be acknowledged. First, different treatment strategies in the study subjects may influence the results of RNA expression levels. Second, further validation of those differentially expressed mRNAs, IncRNAs and miRNAs in a larger cohort is still needed to confirm our preliminary results.

In conclusion, the findings of this study demonstrate a comprehensive expression profile of IncRNAs in SLE with concurrent integrated expression of miRNAs and mRNAs and imply potential regulatory functions of IncRNAs, miRNAs and mRNAs which are implicated in the development and pathogenesis of SLE.

\section{Acknowledgments}

Qin Zhang and Yan Liang contributed equally to this work and should be considered co-first authors.

This work was supported by grants from the National Natural Science Foundation of China (81673258, 81573222, 81473058 ) and Anhui Province Natural Science Foundation (1608085MH219).

\section{Conflict of interest}

The authors declare no conflict of interest.

\section{References}

1. Grammatikos AP, Kyttaris VC, Kis-Toth K, et al. A T cell gene expression panel for the diagnosis and monitoring of disease activity in patients with systemic lupus erythematosus. Clin Immunol 2014; 150: 192-200.
2. Kang SC, Hwang SJ, Chang YS, Chou CT, Tsai CY. Characteristics of comorbidities and costs among patients who died from systemic lupus erythematosus in Taiwan. Arch Med Sci 2012; 8: 690-6.

3. Alexander JJ, Saxena AK, Bao L, Jacob A, Haas M, Quigg RJ. Prominent renal expression of a murine leukemia retrovirus in experimental systemic lupus erythematosus. J Am Soc Nephrol 2002; 13: 2869-77.

4. Pignatelli M, Vilella AJ, Muffato M, et al. ncRNA orthologies in the vertebrate lineage. Database (Oxford) 2016; 2016: pii: bav127.

5. Shvedova AA, Yanamala N, Kisin ER, Khailullin TO, Birch ME, Fatkhutdinova LM. Integrated analysis of dysregulated ncRNA and mRNA expression profiles in humans exposed to carbon nanotubes. PLoS One 2016; 11: e0150628.

6. Ying L, Huang Y, Chen H, et al. Downregulated MEG3 activates autophagy and increases cell proliferation in bladder cancer. Mol Biosyst 2013; 9: 407-11.

7. Kang K, Huang YH, Li HP, Guo SM. Expression of UCA1 and MALAT1 long-chain non-coding RNAs in esophageal squamous cell carcinoma tissues is predictive of patient prognosis. Arch Med Sci 2018; 14: 752-59.

8. Wu Y, Zhang F, Ma J, et al. Association of large intergenic noncoding RNA expression with disease activity and organ damage in systemic lupus erythematosus. Arthritis Res Ther 2015; 17: 131.

9. Wu GC. Immunoregulation function of long noncoding RNA in rheumatic diseases. Chin J Dis Control Prev 2016; 20: 1165-71.

10. Kino T, Hurt DE, Ichijo T, Nader N, Chrousos GP. Noncoding RNA gas 5 is a growth arrest- and starvation-associated repressor of the glucocorticoid receptor. Sci Signal 2010; 3: ra8.

11. Wu GC, Li J, Leng RX, et al. Identification of long noncoding RNAs GAS5, linc0597 and Inc-DC in plasma as novel biomarkers for systemic lupus erythematosus. Oncotarget 2017; 8: 23650-63.

12. Sliwinska A, Kasinska MA, Drzewoski J. MicroRNAs and metabolic disorders - where are we heading? Arch Med Sci 2017; 13: 885-96.

13. Sun XG, Tao JH, Xiang N, et al. Negative correlation between miR-326 and Ets-1 in regulatory T cells from new-onset SLE patients. Inflammation 2016; 39: 822-9.

14. Kaga H, Komatsuda A, Omokawa A, et al. Downregulated expression of miR-155, miR-17, and miR-181b, and upregulated expression of activation-induced cytidine deaminase and interferon-alpha in PBMCs from patients with SLE. Mod Rheumatol 2015; 25: 865-70.

15. Wang $\mathrm{Y}$, Liang J, Qin $\mathrm{H}$, et al. Elevated expression of miR-142-3p is related to the proinflammatory function of monocyte-derived dendritic cells in SLE. Arthritis Res Ther 2016; 18: 263.

16. Chaussabel D, Pascual V, Banchereau J. Assessing the human immune system through blood transcriptomics. BMC Biol 2010; 8: 84.

17. Wang Z, Gerstein M, Snyder M. RNA-Seq: a revolutionary tool for transcriptomics. Nat Rev Genet 2009; 10: 57-63.

18. Stone RC, Du P, Feng D, et al. RNA-Seq for enrichment and analysis of IRF5 transcript expression in SLE. PLoS One 2013; 8: e54487.

19. Zhang Z, Shi L, Dawany N, Kelsen J, Petri MA, Sullivan KE. H3K4 tri-methylation breadth at transcription start sites impacts the transcriptome of systemic lupus erythematosus. Clin Epigenetics 2016; 8: 14.

20. Hochberg MC. Updating the American College of Rheumatology revised criteria for the classification of sys- 
temic lupus erythematosus. Arthritis Rheum 1997; 40: 1725.

21. Ibanez D, Urowitz MB, Gladman DD. Summarizing disease features over time: I. Adjusted mean SLEDAI derivation and application to an index of disease activity in lupus. J Rheumatol 2003; 30: 1977-82.

22. Hsu SD, Lin FM, Wu WY, et al. miRTarBase: a database curates experimentally validated microRNA-target interactions. Nucleic Acids Res 2011; 39: D163-9.

23. Gene Ontology C. The Gene Ontology (GO) project in 2006. Nucleic Acids Res 2006; 34: D322-6.

24. Chen W, Liu BY, Zhang X, et al. Identification of differentially expressed genes in salivary adenoid cystic carcinoma cells associated with metastasis. Arch Med Sci 2016; 12: 881-8.

25. Draghici S, Khatri P, Tarca AL, et al. A systems biology approach for pathway level analysis. Genome Res 2007; 17: 1537-45.

26. Ma H, Zhang S, Xu Y, Zhang R, Zhang X. Analysis of differentially expressed microRNA of TNF-alpha-stimulated mesenchymal stem cells and exosomes from thei culture supernatant. Arch Med Sci 2018; 14: 1102-11.

27. Skretkowicz J, Baranska M, Kaczorowska A, RychlikSych M. Genetic polymorphisms of CYP2D6 oxidation in patients with systemic lupus erythematosus. Arch Med Sci 2011; 7: 864-9.

28. Li J, Lin SY, Lv YB, Tang HM, Peng F. Association study of MMP-9 -1562C/T gene polymorphism with susceptibility to multiple autoimmune diseases: a meta-analysis. Arch Med Res 2017; 48: 105-12.

29. Cheng YY, Sheng YJ, Chang Y, et al. Increased expression of IL-28RA mRNA in peripheral blood mononuclear cells from patients with systemic lupus erythematosus. Clin Rheumatol 2015; 34: 1807-11.

30. Nawrocki MJ, Majewski D, Puszczewicz M, Jagodzinski PP. Decreased mRNA expression levels of DNA methyltransferases type 1 and $3 \mathrm{~A}$ in systemic lupus erythematosus. Rheumatol Int 2017; 37: 775-83.

31. Zhang HJ, Wei QF, Wang SJ, et al. LnCRNA HOTAIR alleviates rheumatoid arthritis by targeting miR-138 and inactivating NF-kappaB pathway. Int Immunopharmacol 2017; 50: 283-90.

32. Webb R, Merrill JT, Kelly JA, et al. A polymorphism within IL21R confers risk for systemic lupus erythematosus. Arthritis Rheum 2009; 60: 2402-7.

33. Paradowska-Gorycka A, Sowinska A, Stypinska B, et al. Impact of the IL-17F, IL-23 and IL-23R on susceptibility and phenotype of systemic lupus erythematosus. Autoimmunity 2016; 49: 373-82.

34. Kaiser R, Tang LF, Taylor KE, et al. A polymorphism in TLR2 is associated with arterial thrombosis in a multiethnic population of patients with systemic lupus erythematosus. Arthritis Rheumatol 2014; 66: 1882-7.

35. Dai R, Ahmed SA. MicroRNA, a new paradigm for understanding immunoregulation, inflammation, and autoimmune diseases. Transl Res 2011; 157: 163-79.

36. Tang $\mathrm{D}$, Chen $\mathrm{Y}$, He H, et al. Integrated analysis of mRNA, microRNA and protein in systemic lupus erythematosus-specific induced pluripotent stem cells from urine. BMC Genomics 2016; 17: 488.

37. Luo X, Zhang L, Li M, et al. The role of miR-125b in T lymphocytes in the pathogenesis of systemic lupus erythematosus. Clin Exp Rheumatol 2013; 31: 263-71.

38. Jeffries MA, Sawalha AH. Epigenetics in systemic lupus erythematosus: leading the way for specific therapeutic agents. Int J Clin Rheumtol 2011; 6: 423-39.
39. da Silva Fonseca AM, de Azevedo Silva J, Pancotto JA, et al. Polymorphisms in STK17A gene are associated with systemic lupus erythematosus and its clinical manifestations. Gene 2013; 527: 435-49.

40. Iwata S, Tanaka Y. B-cell subsets, signaling and their roles in secretion of autoantibodies. Lupus 2016; 25 : 850-6.

41. Maresco DL, Blue LE, Culley LL, Kimberly RP, Anderson CL, Theil KS. Localization of FCGR1 encoding Fcgamma receptor class I in primates: molecular evidence for two pericentric inversions during the evolution of human chromosome 1. Cytogenet Cell Genet 1998; 82: 71-4.

42. Brown EE, Edberg JC, Kimberly RP. Fc receptor genes and the systemic lupus erythematosus diathesis. Autoimmunity 2007; 40: 567-81.

43. Oki K, Plonczynski MW, Gomez-Sanchez EP, Gomez-Sanchez CE. YPEL4 modulates HAC15 adrenal cell proliferation and is associated with tumor diameter. Mol Cell Endocrinol 2016; 434: 93-8.

44. Zhang M, Mu H, Lv H, et al. Integrative analysis of genome-wide association studies and gene expression analysis identifies pathways associated with rheumatoid arthritis. Oncotarget 2016; 7: 8580-9.

45. Chihara N, Aranami T, Oki S, et al. Plasmablasts as migratory IgG-producing cells in the pathogenesis of neuromyelitis optica. PLoS One 2013; 8: e83036.

46. Cen H, Zhou M, Leng RX, et al. Genetic interaction between genes involved in NF-kappaB signaling pathway in systemic lupus erythematosus. Mol Immunol 2013; 56: 643-8. 